\title{
Physical performance in recently aged adults after 6 weeks traditional Thai dance: a randomized controlled trial
}

\author{
Taweesak Janyacharoen ${ }^{1-3}$ \\ Maneepun Laophosri ${ }^{2,4}$ \\ Jaturat Kanpittaya ${ }^{3,5}$ \\ Paradee Auvichayapat ${ }^{6}$ \\ Kittisak Sawanyawisuth ${ }^{7}$ \\ 'School of Physical Therapy, Faculty \\ of Associated Medical Science, Khon \\ Kaen University, Khon Kaen, Thailand; \\ ${ }^{2}$ Improvement of Physical Performance and \\ Quality of Life Research Group, Khon Kaen \\ University, Khon Kaen, Thailand; ${ }^{3}$ Back, \\ Neck and Other Joint Pain Research Group, \\ Khon Kaen University, Khon Kaen, Thailand; \\ ${ }^{4}$ Department of Rehabilitation Medicine, \\ Faculty of Medicine, Khon Kaen University, \\ Khon Kaen, Thailand; ${ }^{5}$ Department of \\ Radiology, Faculty of Medicine, Khon \\ Kaen University, Khon Kaen, Thailand; \\ ${ }^{6}$ Department of Physiology, Faculty of \\ Medicine, Khon Kaen University, Khon \\ Kaen, Thailand; 'Department of Medicine, \\ Faculty of Medicine, Khon Kaen University, \\ Khon Kaen, Thailand
}

\section{Video abstract}

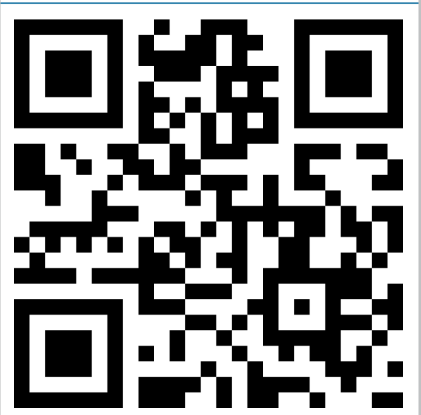

Point your SmartPhone at the code above. If you have a QR code reader the video abstract will appear. Or use: http://dvpr.es/15MQi55

Correspondence: Kittisak Sawanyawisuth Department of Medicine, Faculty of Medicine, Khon Kaen University, Khon Kaen, 40002, Thailand

Tel +66 43363664

Fax +66 43348399

Email kittisak@kku.ac.th
This article was published in the following Dove Press journal:

Clinical Interventions in Aging

20 July 2013

Number of times this article has been viewed

Background: Exercise has been shown to be effective in cardiovascular endurance in the elderly. We studied the effect of Thai dancing on physical performance of Thai elderly.

Methods: This was an open-labeled, randomized intervention study. The Thai dancing group exercised for 40 minutes three times a week for 6 weeks. Physical performance ability was the primary outcome, including a 6-minute walk test (6MWT), five-times sit-to-stand (FTSST), and a sit-and-reach test measured before and after 6 weeks of intervention.

Results: There were 42 subjects enrolled in the study, and 38 female subjects completed ( 20 in Thai dance group, 18 controls), with an average age of $65.8 \pm 5.1$ years. The Thai dance group had significantly better physical performance in all measurements at the end of the study. The 6MWT was longer $(416.7 \pm 58.7$ versus $345.7 \pm 55.1 \mathrm{~m} ; P=0.011)$, FTSST was quicker $(10.2 \pm 1.5$ versus $14.4 \pm 3.3$ seconds; $P<0.001)$, and flexibility was higher $(14.9 \pm 3.5$ versus $11.1 \pm 5.7 \mathrm{~cm} ; P=0.002)$ in the Thai dance group than the control group.

Conclusion: Thai dance can improve physical performance in recently aged (elderly) female adults.

Keywords: recently aged (elderly) adults, exercise, Thai dancing

\section{Introduction}

The world health organization (WHO) defines the elderly in Thailand as people aged over 60 years. ${ }^{1}$ Numbers of elderly people are increasing worldwide due to better health care services and technology. The physical status of the elderly is generally vulnerable and at risk for falling or cardiovascular diseases.

There are several effects of exercise on physical functions, including improvement of disability conditions, ${ }^{2}$ reduction of blood pressure and cholesterol levels, ${ }^{3}$ positive effects on cardiovascular physiology, pain control, quality of life, ${ }^{4-7}$ and fall prevention. $^{2}$

Presently, there are many types of exercise for the elderly; for example, tai chi, qigong, club dancing, yoga, and aerobic exercise. Most of these are other nations' cultural-based exercises. Kulsatitporn et al (2010) showed that enhancement of health and exercise of the elderly should be adjusted according to their lifestyles, their cultural context, traditions, way of living, values, and needs so that the exercise will be included as part of habitual activities performed correctly, individually or as a group, and in a sustainable and suitable manner. ${ }^{8} \mathrm{~A}$ recent review also showed that dancing significantly improves aerobic power, lower body muscle endurance, strength and flexibility, balance, agility, and gait through dancing in the elderly. ${ }^{9}$

Thai dancing is traditional in the art and culture of the Thai people. It is described as a slow continuous dance with Thai classical music with consistent rhythms. The music 
and rhythm of Thai dance is quite similar to tai chi, but Thai dance has more complex posture and focuses on coordination. It is considered as an aerobic dance and has small loads on the knees and ankles. Therefore, it may be suitable for the elderly due to slow rhythm and low joint loads on the lower body. Thai dancing can be incorporated into the community's cultural context, daily life, and the needs of the elderly, which can result in increased/sustained physical capability and quality of life of the elderly.

This study evaluated the effects of classical Thai dance on physical ability of the elderly using recently aged adults as subjects.

\section{Methods}

\section{Trial design (Figure I)}

This was an open-labelled, randomized controlled intervention trial conducted in Thailand.

\section{Participants}

Recently aged Thai adults, where inclusion criteria included an age of over 60 years, independent, were able to walk without any walkers or canes, previously healthy, and had not exercised during the past 2 weeks. Subjects were excluded if they were not able to participate in the entire study or had any diseases or conditions that would limit their being able to perform Thai dance, such as congenital anomalies, stroke, epilepsy, mental diseases, uncontrollable diseases related to heart and blood vessels, obstructive pulmonary disease,

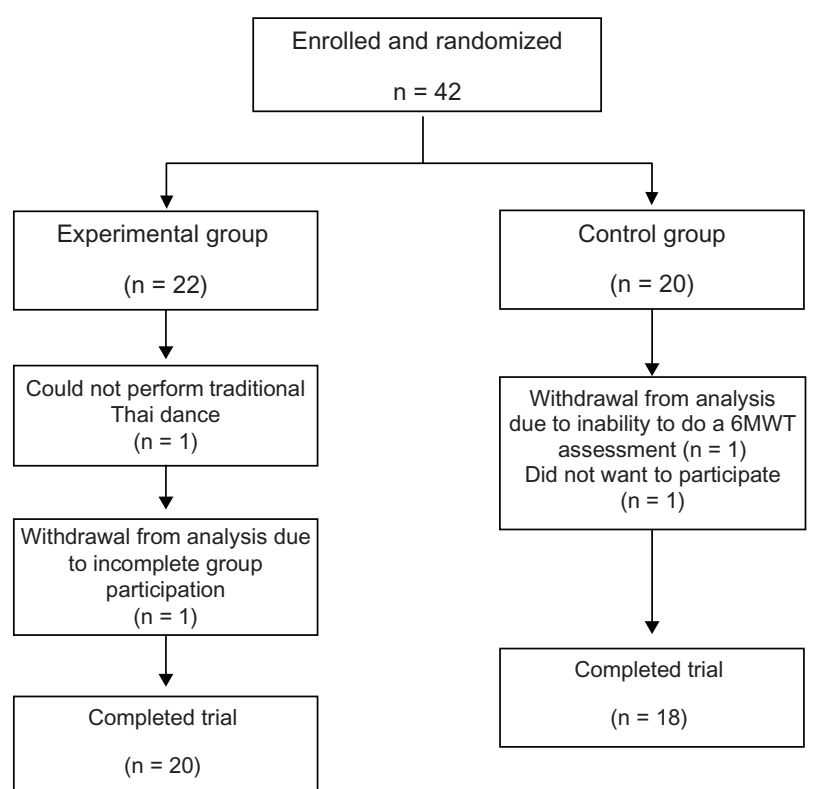

Figure I Recruitment and randomization of subjects; four subjects were excluded prior to the intervention.

Abbreviation: 6MWT, 6-minute walk test. lung diseases, infections, uncontrollable metabolic-related disease, uncontrollable hypertension, and severe pains.

\section{Interventions}

After the subjects signed informed consents, they were randomly divided into a Thai dance group and a control group. The Thai dance group performed a 40-minute, group Thai dance, three times a week for a total of 6 weeks. The control group received instructions on general aerobic exercises in daily life and all subjects were asked to do these exercises for 6 weeks. Subjects were able to withdraw from the study at any time.

\section{Intervention: traditional Thai dance}

The standard traditional Thai dance that was used was composed of songs and postures. Experts in Thai dancing trained the volunteers three times per week for 2 months and then became the Thai dancing leader to facilitate the intervention study. There were both the Thai dance leader and physical therapists taking care of all participants throughout the study period. The dancing leader and physical therapist took care of all participants during the intervention. All subjects performed Thai dance together under observation and care from the leader and physical therapists.

A Thai dance song has a slow rhythm, and the posture changes are also slow but continuous. The postures of Thai dance included arm raising/lowering/bending/stretching, raising right and left arms alternately, raising and lowering legs, stretching knees, standing on toes and flat feet, and turning around (Figure 2 and Thai dancing video http://www.youtube. com/watch? v=batmh4 p2FA).

The program for Thai dance included 5 minutes for warming up or stretching of muscles, dancing the Thai standard dance for 30 minutes, then 5 minutes for relaxing and stretching muscles. The frequency of exercising was three times a week for 6 weeks, 18 times in total.
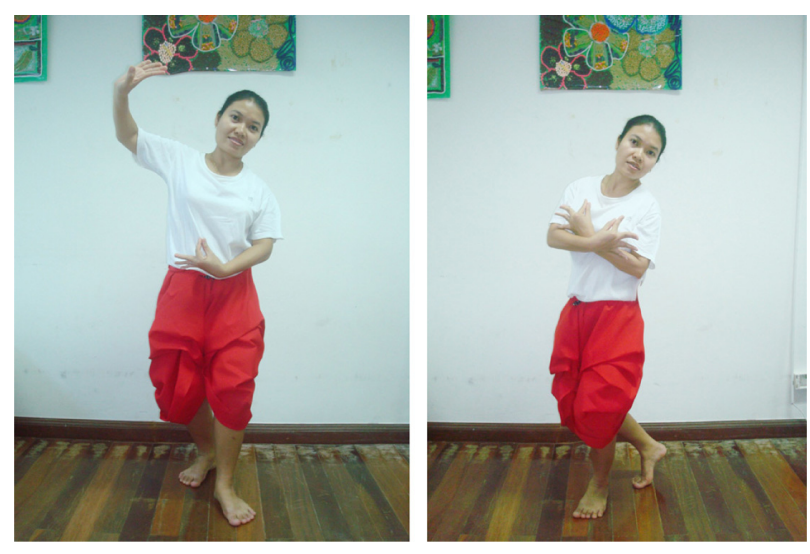

Figure 2 Type of traditional Thai dance. 


\section{Outcomes}

The primary outcomes of the study were in physical performance, namely the strength of heart and blood vessels (6-minute walk test [6MWT], strength of lower limbs, or five-times sit-to-stand test [FTSST], and the flexibility test). All the tests were conducted by the same physical therapist for each test to obtain test validity. The therapists were blinded to the subject's group. All measurements were done at baseline and the end of the study. These physical performance tests have been shown to have good reliability. ${ }^{10-12}$ Other measurements were weight, height, body mass index, heart rate (HR) and blood pressure (BP). The measurement of HR and BP was done after 20 minutes of rest in a calm environment. The device for HR and BP measurement was a Dinamap 1846 SX, USA. All baseline characteristics were recorded before and after the study intervention. Details of each physical performance are provided below.

\section{MWT}

Cardiovascular endurance of the heart and blood vessels was measured by a $6 \mathrm{MWT} .{ }^{13} \mathrm{In}$ the test, the subject walked in a square for 6 minutes continuously for the longest distance possible, twice. The longest distance was measured and recorded.

\section{FTSST}

Lower-limb strength was measured by the FTSST test to determine the strength of the muscles of the lower limbs. The test was easy to perform and is generally used to test the elderly because it imitates daily activities like sitting in a chair or using the toilet. ${ }^{14-16}$ The subject was asked to sit on a $43 \mathrm{~cm}$ high and $47.5 \mathrm{~cm}$ deep chair (or adjusted to fit each subject individually) by leaning their back on the backrest, arms folded on the chest, hip joints at 90 degrees, ankles slightly behind knee joints, and both feet flat on the floor. The subject was asked to rise and sit as quickly as possible five times. The last time, the subject leaned against the backrest of the seat. The subject repeated the test two times, and the shortest time spent was recorded. ${ }^{14}$

\section{Flexibility}

Flexibility was checked by the sit-and-reach test using a trunk flexometer. The subject sat stretching both legs, bending their body, and extending both hands forward as far as possible along the scale. ${ }^{17}$ The test was duplicated, and the best result was recorded. Instruction for the flexibility is detailed below and used a trunk flexometer which is easy for elderly to use.

\section{Sample size calculation}

The primary outcome in this study was the 6MWT. The sample size was calculated based on a previous study. ${ }^{6}$ A 12-week aerobic dance program improved the 6MWT with the effect size of 33.79 and the standard deviation of 39.38 in the intervention group compared with the control group. ${ }^{6}$ Thai dance may improve the 6MWT by mean difference of 0.8 $(80 \%)$ with $80 \%$ power at alpha level of 0.05 and a dropout rate of $20 \%$, a sample size of 52 , and 45 subjects in each group (95 subjects in total). Due to overwhelming response from the social centers, a total of 40 subjects or 20 subjects per group were enrolled.

\section{Ethics approval}

This research was approved by the Human Research Ethics Committee, Khon Kaen University based on the Declaration of Helsinki and Good Clinical Practices (ICHGCP) Number HE542308.

\section{Statistical analysis}

Results are presented as the means \pm standard deviation. 6MWT, FTSST, and flexibility were compared by repeated measures analysis of variance between two groups. Paired $t$-tests were used to compare the differences of all outcome variables between the baseline and the end of study in each group. Analyses were performed with SPSS 17.0. A $P$-value $<0.05$ was considered statistically significant.

\section{Results}

There were 42 subjects enrolled in the study. Of those, 22 subjects (21 female and one male) and 20 subjects (19 female and one male) were in the Thai dance and control group, respectively. Four subjects were withdrawn from the study prior to the intervention: both men because they were unable to perform Thai dance or not willing to participate in the control group, one woman in the Thai dance group could not complete the study protocol, and one woman in the control group could not perform the 6MWT due to pain. In total, 20 female subjects in the Thai dance group and 18 female subjects in the control group completed the study. All baseline variables of both Thai dance and control groups were comparable (Table 1). All 38 female subjects completed the study protocol.

\section{Outcome variables}

All outcome variables were statistically significantly different after the intervention in the Thai dance group (Table 2). The 6MWT increased $(P=0.013)$, the FTSST decreased 
Table I Anthropometric and baseline characteristics of subjects

\begin{tabular}{lcc}
\hline Variables & $\begin{array}{c}\text { Control group } \\
\mathbf{n = ~} \mathbf{~} \mathbf{8}\end{array}$ & $\begin{array}{c}\text { Experimental group } \\
\mathbf{n = 2 0}\end{array}$ \\
\hline Age (years) & $66.8 \pm 6.0$ & $64.9 \pm 4.0$ \\
Weight $(\mathrm{kg})$ & $59.1 \pm \mathrm{II} . \mathrm{I}$ & $59.7 \pm 8.2$ \\
Height $(\mathrm{cm})$ & $153.3 \pm 5.1$ & $153.4 \pm 5.4$ \\
BMI & $25.6 \pm 4.4$ & $25.5 \pm 3.1$ \\
SBP $(\mathrm{mmHg})$ & $135.3 \pm 16.2$ & $142.0 \pm 19.3$ \\
DBP $(\mathrm{mmHg})$ & $73.0 \pm 7.4$ & $83.1 \pm 13.1$ \\
HR (beats/minute) & $74.2 \pm 1 \mathrm{II} .4$ & $76.4 \pm 1 \mathrm{I} .4$ \\
\hline
\end{tabular}

Notes: All subjects were female; values are mean \pm standard deviation.

Abbreviations: BMI, body mass index; SBP, systolic blood pressure; DBP, diastolic blood pressure; HR, heart rate.

$(P<0.001)$, and the flexibility increased $(P=0.001)$ from the baseline in the Thai dance group. There was no significant improvement in any of the outcome variables in the control group (Table 2).

All outcome variables in the Thai dance group were significantly different from the control group at the end of the study (Table 2 ). The $6 \mathrm{MWT}$ was longer $(416.7 \pm 58.7$ versus $345.7 \pm 55.1 \mathrm{~m} ; P=0.011)$, FTSST was quicker $(10.2 \pm 1.5$ versus $14.4 \pm 3.3 \mathrm{~s} ; P<0.001)$, and the flexibility was higher $(14.9 \pm 3.5$ versus $11.1 \pm 5.7 \mathrm{~cm} ; P=0.002)$ in the Thai dance group than the control group.

\section{Discussion}

The present study demonstrated that Thai dance significantly improved a subject's physical performance in female Thai recently aged adults with mean age of 65 years. All three physical performance parameters were significantly improved after 6 weeks of Thai dance and also significantly different from the baseline (Table 2), whereas the control group did not show any differences. The 6MWT indicates endurance, FTSST indicates balance and coordination, and the flexibility test improves flexibility.

The young older adults in this study were able to walk at least $66 \mathrm{~m}$ after the 6 weeks of Thai dance. Previous studies showed that it would be a clinically significant improvement if elderly with heart failure or stroke were able to walk more than $40-50 \mathrm{~m} \cdot{ }^{18,19}$ A reduction of at least 2.3 seconds in
FTSST is considered a statistically significant improvement. ${ }^{20}$ The Thai dance subjects were able to sit and stand faster after intervention by 2.7 seconds (Table 2). Trunk flexibility has been shown to be associated with arterial stiffness and may increase the risk of stroke and coronary heart disease in the elderly. ${ }^{21,22}$ Having flexibility less than $26 \mathrm{~cm}$ in adults aged more than 60 years was associated with poor arterial stiffness. ${ }^{22}$ Even though Thai dance improved flexibility, it seemed that the subjects may have some degree of arterial stiffness. Even in the Thai dance group, the sit and reach distance was just $14.9 \mathrm{~cm}$.

Thai dance used in this study followed the protocol for an aerobic exercise. The period of Thai dancing exercise was 40 minutes 3 times per week. After 6 weeks of the exercise, physical performances of our recently aged adult females significantly improved. These findings are supported by three other studies. One study showed that aerobic dancing of the elderly three times per week for 8 weeks improved physical functions. ${ }^{6}$ The other two studies showed that cultural dancing improved physical fitness of the elderly; Turkey's folk dance (three times a week for 8 weeks) and Korean folk dance (6 months showed improvement). ${ }^{23,24}$ Another benefit for aerobic dancing exercise in healthy elderly women was the reduction in fall risks. ${ }^{25}$

There are some limitations in this study. All subjects are independent recently aged female Thai elderly. The results may not be universal for all elderly. Thai dance is easy for Thai females to perform. Most people in Thailand study traditional Thai dance at an early age in school, and Thai dance represents the female sex which resulted in no participation of Thai men in this study, which was also the case in a study of Korean dance. ${ }^{25}$ Performing in a group may improve group participation though. Thai dance may be suitable for people who are interested in Thai culture. Even though numbers of subjects in this study are quite small and the study duration is short, physical performances are statistically improved by participating in the Thai dance. Finally, further study comparing Thai dance with other exercise methods such as tai chi and yoga will be worthwhile.

Table 2 Physical performances of controls and subjects in Thai dance group at week 0 and the end of study

\begin{tabular}{lccccc}
\hline Variables & \multicolumn{2}{c}{ Control group $(\mathbf{n}=\mathbf{~ 1 8})$} & & \multicolumn{2}{c}{ Thai dance group $(\mathbf{n}=\mathbf{2 0})$} \\
\cline { 2 - 3 } & At week 0 & At week 6 & & At week 0 & At week 6 \\
\hline 6-minute walk test $(\mathrm{m})$ & $348.0 \pm 51.2$ & $345.7 \pm 55.1$ & & $360.1 \pm 59.2$ & $416.7 \pm 58.7^{\mathrm{a}, \mathrm{b}}$ \\
Five-time sit-to-stand (seconds) & $14.8 \pm 3.7$ & $14.4 \pm 3.3$ & & $12.9 \pm 2.1$ & $10.2 \pm 1.5^{\mathrm{a}, \mathrm{b}}$ \\
Flexibility test $(\mathrm{cm})$ & $10.7 \pm 5.7$ & $11.1 \pm 5.7$ & & $10.4 \pm 3.8$ & $14.9 \pm 3.5^{\mathrm{a}, \mathrm{b}}$ \\
\hline
\end{tabular}

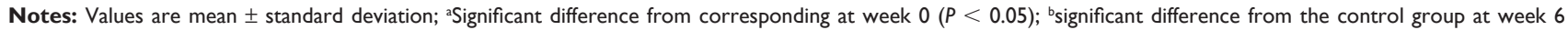
$(P<0.05)$ by repeated measured analysis of variance. 
In conclusion, Thai dance can improve physical performance of female recently aged (elderly) Thai adults.

\section{Acknowledgments}

This study was supported by the Graduate School, the Faculty of Associated Medical Sciences, the Faculty of Medicine, and the Research Group in Physical Performance and Quality of Life (IPQ), Khon Kaen University. The authors wish to acknowledge the support of the Khon Kaen University Publication Clinic, Research and Technology Transfer Affairs, Khon Kaen University for their assistance.

\section{Disclosure}

The authors report no conflicts of interest in this work.

\section{References}

1. Bongaarts J, Zimmer Z. Living arrangements of older adults in the developing world: an analysis of demographic and health survey household surveys. J Gerontol B Psychol Sci Soc Sci. 2002;57(3): S145-S157.

2. Crocker T, Forster A, Young J, et al. Physical rehabilitation for older people in long-term care. Cochrane Database Syst Rev. 2013;CD004294. pub3.

3. Sousa N, Mendes R, Abrantes C, Sampaio J, Oliveira J. Long-term effects of aerobic training versus combined aerobic and resistance training in modifying cardiovascular disease risk factors in healthy elderly men. Geriatr Gerontol Int. Epub February 26, 2013.

4. Penninx BW, Rejeski WJ, Pandya J, et al. Exercise and depressive symptoms: a comparison of aerobic and resistance exercise effects on emotional and physical function in older persons with high and low depressive symptomatology. J Gerontol B Psychol Sci Soc Sci. 2002; 57(2):P124-P132.

5. Mazzeo R, Tanaka H. Exercise prescription for the elderly: current recommendations. Sports Med. 2001;31(11):809-818.

6. Hui E, Chui BT, Woo J. Effects of dance on physical and psychological well-being in older persons. Arch Gerontol Geriatr. 2009;49(1): e45-e50.

7. Karacan S. Effects of long-term aerobic exercise on physical fitness and postmenopausal symptoms with menopausal rating scale. Sci Sports. 2010;25:39-46.

8. Kulsatitporn S, Ariyapitipan T. Modified the transtheoretical model in health behavior modification for prevention of chronic disease in rural community dwelling elderly: a case study in Dongruay district, Damnoensaduak, Ratchburi. NRCT. 2008:1-7. Thai.

9. Keogh JW, Kilding A, Pidgeon P, Ashley L, Gillis D. Physical benefits of dancing for healthy older adults: a review. J Aging Phys Act. 2009; 17(4):479-500.
10. Nasuti G, Stuart-Hill L, Temple VA. The Six-Minute Walk Test for adults with intellectual disability: a study of validity and reliability. J Intellect Dev Disabil. 2013;38(1):31-38.

11. Goldberg A, Chavis M, Watkins J, Wilson T. The five-times-sit-to-stand test: validity, reliability and detectable change in older females. Aging Clin Exp Res. 2012;24(4):339-344.

12. Jones MA, Stratton G, Reilly T, et al. Measurement error associated with spinal mobility measures in children with and without low-back pain. Acta Paediatr. 2002;91(12):1339-1343.

13. Swisher A, Goldfarb A. Use of the Six-Minute Walk/Run Test to predict peak oxygen consumption in older adults. Cardiopulm Phys Ther J. 1998;9(3):3-5.

14. Whitney SL, Wrisley DM, Marchetti GF, Gee MA, Redfern MS, Furman JM. Clinical measurement of sit-to-stand performance in people with balance disorders: validity of data for the Five-Times-Sit-to-Stand Test. Phys Ther. 2005;85:1034-1045.

15. Lord SR, Murray SM, Chapman K, Munro B, Tiedemann A. Sit-to-stand performance depends on sensation, speed, balance, and psychological status in addition to strength in older people. J Gerontol A Biol Sci Med Sci. 2002;57(8):M539-M543.

16. Bohannon RW. Aternatives for measuring knee extension strength of the elderly at home. Clin Rehabil. 1998;12(5):434-440.

17. Jones CJ, Rikli RE, Beam WC. A 30-s chair-stand test as a measure of lower body strength in community-residing older adults. Res $Q$ Exerc Sport. 1999;70(2):113-119.

18. Perera S, Mody S, Woodman R, Studenski S. Meaningful change and responsiveness in common physical performance measures in older adults. J Am Geriatr Soc. 2006;54(5):743-749.

19. O'Keeffe ST, Lye M, Donnellan C, Carmichael DN. Reproducibility and responsiveness of quality of life assessment and six minute walk test in elderly heart failure patients. Heart. 1998;80(4):377-382.

20. Meretta BM, Whitney SL, Marchetti GF, Sparto PJ, Muirhead RJ. The five times sit to stand test: Responsiveness to change and concurrent validity in adults undergoing vestibular rehabilitation. $J$ Vestib Res. 2006;16(4-5):233-243.

21. Sutton-Tyrrell K, Najjar SS, Boudreau RM, et al. Elevated aortic pulse wave velocity, a marker of arterial stiffness, predicts cardiovascular events in well-functioning older adults. Circulation. 2005;111(25): 3384-3390.

22. Yamamoto K, Kawano H, Gando Y, et al. Poor trunk flexibility is associated with arterial stiffening. Am J Physiol Heart Circ Physiol. 2009;297(4):H1314-H1318.

23. Eyigor S, Karapolat H, Durmaz B, Ibisoglu U, Cakir S. A randomized controlled trial of Turkish folklore dance on the physical performance, balance, depression and quality of life in older women. Arch Gerontol Geriatr. 2009;48(1):84-88.

24. Song R, June KJ, Kim CG, Jeon MY. Comparisons of motivation, health behaviors, and functional status among elders in residential homes in Korea. Public Health Nurs. 2004;21(4):361-371.

25. Shigematsu R, Chang M, Yabushita N, et al. Dance-based aerobic exercise may improve indices of falling risk in older women. Age Ageing. 2002;31(4):261-266.
Clinical Interventions in Aging

\section{Publish your work in this journal}

Clinical Interventions in Aging is an international, peer-reviewed journal focusing on evidence-based reports on the value or lack thereof of treatments intended to prevent or delay the onset of maladaptive correlates of aging in human beings. This journal is indexed on PubMed Central, MedLine, the American Chemical Society's 'Chemical Abstracts

\section{Dovepress}

Service' (CAS), Scopus and the Elsevier Bibliographic databases. The manuscript management system is completely online and includes a very quick and fair peer-review system, which is all easy to use. Visit http://www.dovepress.com/testimonials.php to read real quotes from published authors. 